\title{
Sitagliptin on Carotid Intima-Media Thickness in Type 2 Diabetes Mellitus Patients and Anemia: A Subgroup Analysis of the PROLOGUE Study
}

\author{
Zhengri Lu $\mathbb{D}^{1}{ }^{1}$ Genshan Ma, ${ }^{1,2}$ and Lijuan Chen $\mathbb{D}^{1,2}$ \\ ${ }^{1}$ School of Medicine, Southeast University, Nanjing, China \\ ${ }^{2}$ Department of Cardiology, Zhongda Hospital, Southeast University, Nanjing, China \\ Correspondence should be addressed to Lijuan Chen; chenlijuan15@outlook.com
}

Received 30 December 2019; Revised 7 April 2020; Accepted 11 April 2020; Published 11 May 2020

Academic Editor: Cristina Contreras

Copyright (c) 2020 Zhengri Lu et al. This is an open access article distributed under the Creative Commons Attribution License, which permits unrestricted use, distribution, and reproduction in any medium, provided the original work is properly cited.

\begin{abstract}
Introduction. Randomized clinical trials have not shown an additional clinical benefit of sitagliptin treatment over conventional treatment alone. However, studies of sitagliptin treatment have not examined the relationship between anemia and treatment group outcomes. Methods. The PROLOGUE study is a prospective clinical trial of 442 participants with type 2 diabetes mellitus (T2DM) randomized to sitagliptin treatment or conventional treatment which showed no treatment differences [Estimated mean ( \pm standard error) common carotid intima-media thickness (CIMT) was $0.827 \pm 0.007 \mathrm{~mm}$ and $0.837 \pm 0.007 \mathrm{~mm}$, respectively, with a mean difference of $-0.009 \mathrm{~mm}(97.2 \% \mathrm{CI}-0.028$ to $0.011, p=0.309)$ at 24 mo of follow-up]. This is a post hoc subanalysis using data obtained from the PROLOGUE study; the study population was divided into anemic groups ( $n=94$ ) and nonanemic group $(n=343)$ based on hemoglobin level. And we analyzed for the changes in each CIMT parameter from baseline to 24 months in subgroups. Results. The treatment group difference in baseline-adjusted mean common carotid artery(CCA-) IMT at 24 months was $-0.003 \mathrm{~mm}(95 \%$ CI -0.022 to $0.015, p=0.718)$ in the nonanemic subgroup and $-0.007 \mathrm{~mm}$ ( $95 \%$ CI -0.043 to $0.030, p=0.724$ ) in the anemic subgroup. Although there were no significant differences in the other CIMT parameters between the treatment groups in the anemic subgroup, the changes in mean and max ICA-IMT at 24 months in the nonanemic subgroup were significantly lower in the sitagliptin group than the conventional group [ $-0.104 \mathrm{~mm}(95 \% \mathrm{CI}$ -0.182 to -0.026$), p=0.009$ and $-0.142 \mathrm{~mm}(-0.252$ to -0.033$), p=0.011$, respectively]. Conclusion. These data suggest that nonanemia may indicate a potentially large subgroup of those with T2DM patients that sitagliptin therapy has a better antiatherosclerotic effect than conventional therapy. Further research is needed to confirm these preliminary observations.
\end{abstract}

\section{Introduction}

Atherosclerosis is an inflammatory disease involving the interaction of genetic and environmental factors. It is usually caused by hypertension, hyperlipidemia, diabetes, smoking, and unhealthy diet, which is the leading cause of vascular disease globally. Among them, diabetes mellitus is not only a disorder of glucose metabolism but is also considered to be a high-risk disease that is causing atherosclerosis. A prospective cohort study has shown that the lifetime risk of vascular death in diabetic patients without previous coronary heart disease (CHD) is as high as the risk of CHD only [1]. Therefore, active and effective interventions are needed, including dietary change, physical exercise, and medication to reduce the prevalence of diabetes.

The carotid intima-media thickness (CIMT) is a surrogate marker of atherosclerosis, which is the combined thickness of the tunica intima and media of a circulatory vessel detectable noninvasively with ultrasonographic techniques [2]. On the one hand, CIMT is directly associated with the risk of myocardial infarction and stroke and is considered to be an effective tool for early diagnosis of atherosclerosis $[3,4]$. Some studies suggest that the progression of carotid IMT in coronary artery disease (CAD) can be used to predict coronary events and related mortality [5-7]. The link between CIMT and CAD may be related to inflammation, 
which is recognized to play a critical role in the pathogenesis of atherosclerosis [8]. It has been recognized that the pathogenesis of increased CIMT and CAD are both related to atherosclerosis. These findings emphasize the importance of recognizing and managing the early stages of atherosclerosis for effective prevention of CAD. On the other hand, assessing the efficacy of drugs for diabetes is an active area of therapeutic research in metabolic diseases. Some studies have attempted to evaluate the effects of various drugs on CIMT changes. A systematic review demonstrated that statins can reduce CIMT by lipid decrease [9]. Another meta-analysis suggested that alpha-glucosidase inhibitors (alpha-GIs) can attenuate the CIMT progression in patients with impaired glucose tolerance or type 2 diabetes mellitus (T2DM) [10]. However, to date, there are less data on dipeptidyl peptidase-4 (DPP-4) inhibitors and glucagon-like peptide-1 (GLP-1) receptor agonists associated with CIMT progression. A meta-analysis of 5 studies revealed that there was no statistically significant decrease in IMT by GLP-1 based therapies [11]. DPP-4 inhibitors are a class of antihyperglycemic drugs that can effectively increase the concentration of insulin and control blood glucose levels. In addition, DPP-4 inhibitors may have additional effects beyond blood glucose control, such as antiatherosclerotic effects $[12,13]$. Several researches using animal models have confirmed that DPP-4 inhibitors significantly suppressed atherosclerotic lesions mainly through the actions of GLP-1 and glucose-dependent insulinotropic polypeptide (GIP) [14-18]. In addition, clinical studies have also demonstrated the anti-inflammatory and antiatherosclerotic effects of DPP-4 inhibitors [19, 20]. However, some large-scale clinical trials have found that the DPP-4 inhibitors neither increase nor decrease the incidence of cardiovascular events [21-23]. In addition, some studies have shown that DPP-4 inhibitors can reduce the CIMT increase [24, 25]. Sitagliptin (a DPP-4 inhibitor) and liraglutide (a GLP-1 receptor agonist) treatment improved arterial stiffness by reducing oxidative stress in T2DM patients $[26,27]$. But the PROLOGUE trial did not find that sitagliptin showed an additional effect in inhibiting the progression of CIMT. Therefore, the antiatherosclerotic effect of DPP-4 inhibitors has not been fully elucidated.

Diabetes patients are often accompanied by anemia, which is associated with an increased risk of adverse cardiovascular events and kidney disease [28-30]. The prevalence of anemia in patients with diabetic nephropathy is higher than that with other types of chronic kidney disease (CKD), and the severity of anemia is increasing gradually with the deterioration of renal function. Hemoglobin concentration in patients with diabetes continued to decrease significantly even without nephropathy [31]. Although previous studies have reported abnormal hemoglobin concentrations in patients with diabetes $[31,32]$, other studies have also shown an association between anemia and cardiovascular disease (CVD) in patients with diabetes mellitus and in those with chronic kidney disease (CKD) $[33,34]$. But the relationship between anemia and subclinical atherosclerosis markers in patients with T2DM remains somewhat elusive. In general, we rarely consider anemia as a factor in the development of atherosclerosis in patients with diabetes. Although the prospective randomized clinical trial of sitagliptin has not shown other clinical benefits, the effects of baseline anemia events on CIMT after sitagliptin treatment are unclear. We used data from the PROLOGUE study to test the hypothesis that certain patients in low- and high-risk subgroups were more likely to benefit from sitagliptin.

\section{Methods}

2.1. Study Design. This is a post hoc analysis using data from the PROLOGUE study, a multicenter, randomized, prospective, open-label, blinded endpoint trial carried out at 48 institutions in Japan that evaluated 442 patients with T2DM between June 2011 and September 2012 [35]. The inclusion criteria were age $\geq 30$ years and presence of T2DM with HbA1c (JDS) 6.2-9.4\% (JDS indicates Japan Diabetes Society value that is expressed as $0.4 \%$ lower than National Glycohemoglobin Standardization Program value) despite treatment with diet, exercise, and/or conventional antidiabetic agents (except incretin-related therapy) [36]. Patients with the administration of DPP-4 inhibitors and/or GLP-1 analogues before randomization and heart failure with New York Heart Association functional class III or IV were excluded. Study inclusion and exclusion criteria have been published previously. There were two treatment groups in PROLOGUE: sitagliptin treatment ("sitagliptin group", $n=222$ ) and conventional glucose-lowering treatment ("conventional group", $n=220$ ). The PROLOGUE study primary endpoint was the change in mean common carotid artery- (CCA-) IMT at 24 months after treatment randomization. Other CIMT parameters, including the internal carotid artery- (ICA-) IMT, were secondary endpoints. The study was approved by all participating institutional review boards, and all study participants gave informed consent. The full study protocol can be found in previously published research.

In the post hoc analysis, anemia was defined according to the concentration of hemoglobin, which is the standard index of anemia. According to the World Health Organization (WHO) definition of anemia: hemoglobin $<13 \mathrm{~g} / \mathrm{DL}$ in men and $<12 \mathrm{~g} / \mathrm{DL}$ in women [37]. The study population was divided into the anemic group $(n=94)$ and the nonanemic group $(n=343)$ based on this definition. And we analyzed for the changes in each CIMT parameter from baseline to 24 months in subgroups.

2.2. Statistical Analysis. Normally distributed continuous data were shown as mean \pm standard deviation and compared using a two-tailed unpaired Student's $t$-test. Categorical variables were expressed as frequencies (\%) and compared using the Chi-squared test wherever appropriate. The baselineadjusted means of each parameter were estimated by analysis of covariance with treatment effect and age, sex, statin use, prerandomization treatment type, baseline $\mathrm{HbAlc}$, baseline office systolic blood pressure, baseline maximum IMT, and the baseline value of each parameter as covariates. A twotailed $p$ value $<0.05$ was definitely statistically significant. Statistical analyses were performed with SPSS Statistics Software (version 25.0; IBM SPSS, Armonk, New York, United States of America). 
TABLE 1: Baseline clinical characteristics of the study population.

\begin{tabular}{|c|c|c|c|c|c|c|}
\hline \multirow[b]{2}{*}{ Variable } & \multicolumn{2}{|c|}{ Anemia $(\mathrm{NO}, n=343)$} & \multicolumn{4}{|c|}{ Anemia (YES, $n=94)$} \\
\hline & $\begin{array}{l}\text { Sitagliptin group } \\
\quad(n=175)\end{array}$ & $\begin{array}{l}\text { Conventional } \\
\quad(n=168)\end{array}$ & $P$ & $\begin{array}{l}\text { Sitagliptin group } \\
\quad(n=44)\end{array}$ & $\begin{array}{c}\text { Conventional } \\
\quad(n=50)\end{array}$ & $P$ \\
\hline Mean age (years) & $68.18 \pm 8.87$ & $68.16 \pm 9.34$ & 0.982 & $73.95 \pm 8.27$ & $73.56 \pm 6.95$ & 0.802 \\
\hline Gender (male), $n(\%)$ & $118(67.4 \%)$ & $114(67.9 \%)$ & 0.932 & $25(56.8 \%)$ & $36(72.0 \%)$ & 0.124 \\
\hline BMI $\left(\mathrm{kg} / \mathrm{m}^{2}\right)$ & $25.55 \pm 4.14$ & $25.25 \pm 4.05$ & 0.504 & $24.24 \pm 3.83$ & $23.75 \pm 3.54$ & 0.521 \\
\hline Dyslipidemia (\%) & $132(75.4 \%)$ & $117(69.6 \%)$ & 0.230 & $28(63.6 \%)$ & $30(60.0 \%)$ & 0.717 \\
\hline Cerebral infarction (\%) & $16(9.1 \%)$ & $17(10.1 \%)$ & 0.759 & $4(9.1 \%)$ & $8(16.0 \%)$ & 0.317 \\
\hline Myocardial infarction (\%) & $34(19.4 \%)$ & $38(22.6 \%)$ & 0.468 & $10(22.7 \%)$ & $17(34.0 \%)$ & 0.228 \\
\hline PCI (\%) & $42(24.0 \%)$ & $50(29.8 \%)$ & 0.229 & $16(36.4 \%)$ & $19(38.0 \%)$ & 0.870 \\
\hline CABG (\%) & $16(9.1 \%)$ & $11(6.5 \%)$ & 0.372 & $3(6.8 \%)$ & $5(10.0 \%)$ & 0.581 \\
\hline Chronic heart failure (\%) & $14(8.0 \%)$ & $16(9.5 \%)$ & 0.618 & $1(2.3 \%)$ & $10(20.0 \%)$ & 0.008 \\
\hline Arrhythmia (\%) & $28(16.0 \%)$ & $23(13.7 \%)$ & 0.548 & $4(9.1 \%)$ & $9(18.0 \%)$ & 0.212 \\
\hline Stroke (\%) & $21(12.0 \%)$ & $21(12.5 \%)$ & 0.888 & $5(11.4 \%)$ & $9(18.0 \%)$ & 0.367 \\
\hline SBP $(\mathrm{mmHg})$ & $130.19 \pm 15.76$ & $128.08 \pm 15.73$ & 0.215 & $130.18 \pm 14.87$ & $131.02 \pm 19.10$ & 0.812 \\
\hline DBP (mmHg) & $73.50 \pm 10.65$ & $72.81 \pm 11.20$ & 0.560 & $70.36 \pm 10.70$ & $68.06 \pm 11.80$ & 0.327 \\
\hline HbAlc (\%) & $6.99 \pm 0.69$ & $6.96 \pm 0.54$ & 0.652 & $6.80 \pm 0.36$ & $6.95 \pm 0.59$ & 0.138 \\
\hline FPG (mg/dL) & $140.15 \pm 43.86$ & $137.31 \pm 35.10$ & 0.520 & $130.52 \pm 32.32$ & $126.06 \pm 42.18$ & 0.579 \\
\hline Non-HDL cholesterol (mg/dL) & $126.16 \pm 29.02$ & $125.99 \pm 31.19$ & 0.958 & $107.12 \pm 28.32$ & $103.34 \pm 24.23$ & 0.494 \\
\hline Serum creatinine $(\mathrm{mg} / \mathrm{dL})$ & $0.83 \pm 0.21$ & $0.82 \pm 0.22$ & 0.937 & $0.97 \pm 0.29$ & $0.97 \pm 0.27$ & 0.997 \\
\hline eGFR $\left(\mathrm{mL} / \mathrm{min} / 1.73 \mathrm{~m}^{2}\right)$ & $68.85 \pm 16.66$ & $69.64 \pm 17.55$ & 0.671 & $56.83 \pm 17.57$ & $58.58 \pm 16.23$ & 0.617 \\
\hline Mean CCA IMT (mm) & $0.819 \pm 0.146$ & $0.816 \pm 0.161$ & 0.860 & $0.833 \pm 0.222$ & $0.904 \pm 0.255$ & 0.673 \\
\hline Mean bulb IMT (mm) & $1.060 \pm 0.381$ & $1.129 \pm 0.451$ & 0.182 & $1.330 \pm 0.550$ & $1.173 \pm 0.352$ & 0.154 \\
\hline Mean ICA IMT (mm) & $0.782 \pm 0.279$ & $0.772 \pm 0.319$ & 0.780 & $0.799 \pm 0.246$ & $0.816 \pm 0.268$ & 0.793 \\
\hline Max CCA IMT (mm) & $1.037 \pm 0.197$ & $1.047 \pm 0.215$ & 0.659 & $1.132 \pm 0.316$ & $1.185 \pm 0.370$ & 0.463 \\
\hline Max bulb IMT (mm) & $1.522 \pm 0.570$ & $1.603 \pm 0.677$ & 0.286 & $1.865 \pm 0.843$ & $1.741 \pm 0.537$ & 0.459 \\
\hline Max ICA IMT (mm) & $1.059 \pm 0.375$ & $1.039 \pm 0.440$ & 0.700 & $1.057 \pm 0.333$ & $1.135 \pm 0.397$ & 0.399 \\
\hline Plaque area $\left(\mathrm{mm}^{2}\right)$ & $11.083 \pm 7.311$ & $11.283 \pm 6.656$ & 0.837 & $12.903 \pm 7.992$ & $13.085 \pm 14.233$ & 0.955 \\
\hline Plaque gray scale median & $52.335 \pm 23.765$ & $51.094 \pm 18.747$ & 0.675 & $44.525 \pm 15.908$ & $55.956 \pm 28.407$ & 0.077 \\
\hline
\end{tabular}

Abbreviation: BMI: body mass index; PCI: percutaneous coronary intervention; CABG: coronary artery bypass grafting; SBP: systolic blood pressure; DBP: diastolic blood pressure; FPG: fasting plasma glucose; HDL: high-density lipoprotein; eGFR: estimated glomerular filtration rate; CCA: common carotid artery; IMT: intima-media thickness; ICA: internal carotid artery.

\section{Results}

3.1. Baseline Characteristics of Study Subjects. The baseline demographic and clinical characteristics of the 442 study participants (222 subjects in the sitagliptin group and 220 in the conventional treatment group) have been previously reported. There were no significant differences in terms of the clinical parameters between the two groups. In this post hoc analysis, we compared the baseline demographics and clinical variables in subgroups (Table 1). As shown by the baseline hemoglobin distribution in Figure 1, the hemoglobin concentrations of male and female nonanemic patients were $14.642 \pm 1.030 \mathrm{~g} / \mathrm{DL}$ and $13.366 \pm 0.807 \mathrm{~g} / \mathrm{DL}$, respectively. And the hemoglobin concentrations of male and female anemic patients were $11.944 \pm 0.732 \mathrm{~g} / \mathrm{DL}$ and $10.988 \pm 1.018 \mathrm{~g} /$ $\mathrm{DL}$, respectively. The baseline variables were similar between treatment groups, except more prevalent prior to chronic heart failure in patients treated with conventional in the ane- mic subgroup. The average body mass index, diastolic blood pressure, fasting plasma glucose, and prevalence of dyslipidemia were modestly higher in the nonanemic subgroup. Baseline HbAlc levels in the two subgroups were around 7.0\%. No significant difference in CIMT parameters between the treatment groups in each subgroup.

3.2. Effect of Sitagliptin on Metabolic Factors and Carotid IMT in Subgroups. The effects of sitagliptin on metabolic factors and CIMT have been previously reported. In conclusion, sitagliptin treatment has a more effective hypoglycemic effect than conventional treatment. In this post hoc analysis of the PROLOGUE study, we also found a similar result. The changes in HbAlc at 24 months in the nonanemic subgroup were significantly lower in the sitagliptin group than the conventional group $[-0.146 \mathrm{~mm}(95 \%$ CI -0.282 to $-0.010), p=0.035]$. However, regarding blood pressure, non-HDL cholesterol, serum creatinine, and estimated 


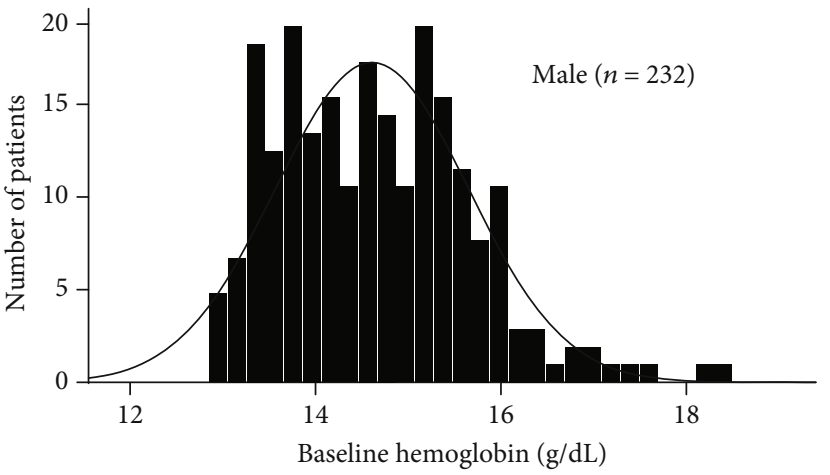

(a)

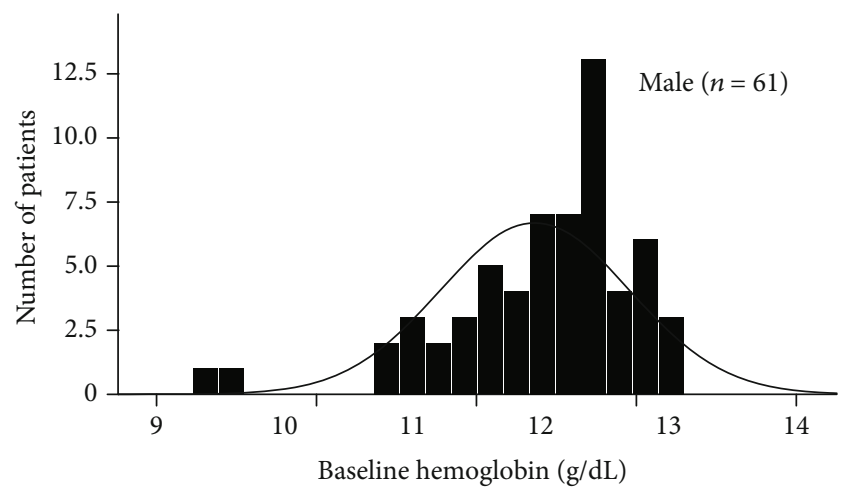

(c)

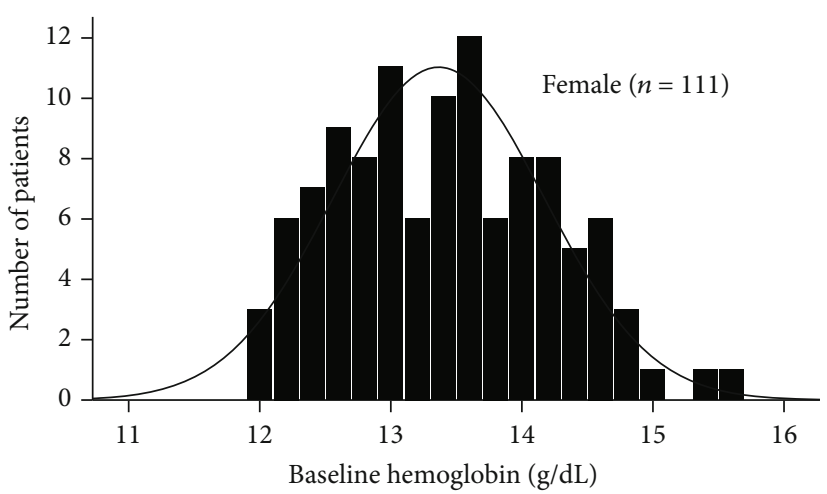

(b)

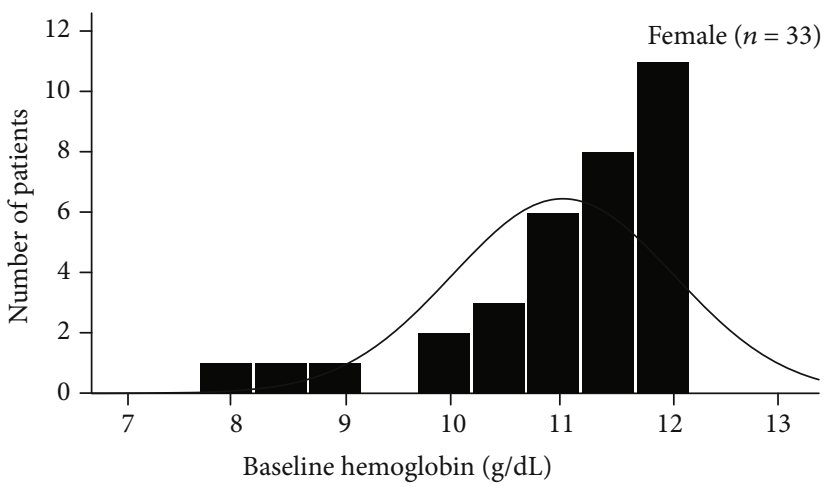

(d)

FIGURE 1: Distribution of the baseline hemoglobin concentrations across the entire cohort of male and female patients. (a) and (b) indicate the number of male and female nonanemic patients, respectively. While (c) and (d) indicate the number of male and female anemic patients, respectively.

glomerular filtration rate (eGFR), there was no significance between treatment group differences in changes from baseline to 24 months. As shown in Figure 2, hemoglobin concentration was slightly higher in patients treated with sitagliptin than in patients treated with conventional in the anemic subgroup $(11.981 \pm 0.180 \mathrm{~g} / \mathrm{DL}$ and $11.473 \pm 0.178 \mathrm{~g} / \mathrm{DL}, p=$ $0.027)$. The treatment group difference in baseline-adjusted mean CCA-IMT at 24 months was $-0.003 \mathrm{~mm}$ (95\% CI -0.022 to $0.015, p=0.718)$ in the nonanemic subgroup and $-0.007 \mathrm{~mm}(95 \% \mathrm{CI}-0.043$ to $0.030, p=0.724)$ in the anemic subgroup (Tables 2 and 3). Although there were no significant differences in the other CIMT parameters between the treatment groups in the anemic subgroup, the changes in mean and max ICA-IMT at 24 months in the nonanemic subgroup were significantly lower in the sitagliptin group than the conventional group $[-0.104 \mathrm{~mm}(95 \% \mathrm{CI}-0.182$ to -0.026$), p=0.009$ and $-0.142 \mathrm{~mm}(-0.252$ to -0.033$)$, $p=0.011$, respectively].

3.3. Effect of Sitagliptin on the Ultrasonic Tissue Characteristics of the Carotid Wall in Subgroups. There were no significant differences in the GSM-Plaque between the two treatment groups in each subgroup at baseline and 24 months (Tables 1-3).

3.4. Antidiabetic and Other Medications in Subgroups during the Study. There were no significant differences in the baseline

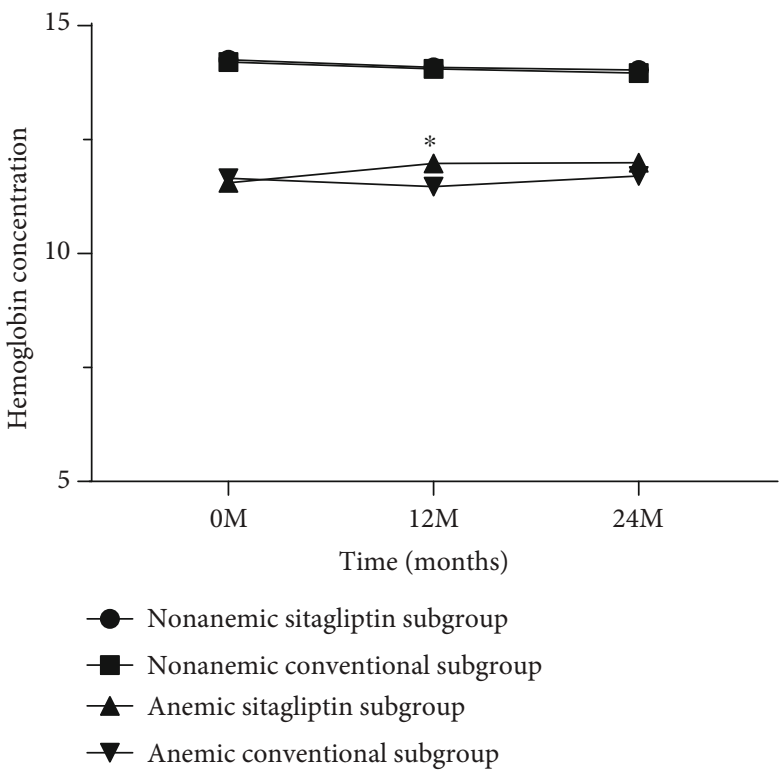

FIgURE 2: Time course of hemoglobin concentration of anemic and nonanemic subgroups during treatment. Graph shows sex-, age- and baseline-adjusted mean ( \pm standard error) at 12 and 24 months. Hemoglobin at 12 months shows a significant difference in the anemic subgroup. ${ }^{*} p=0.027$ vs. conventional subgroup. 


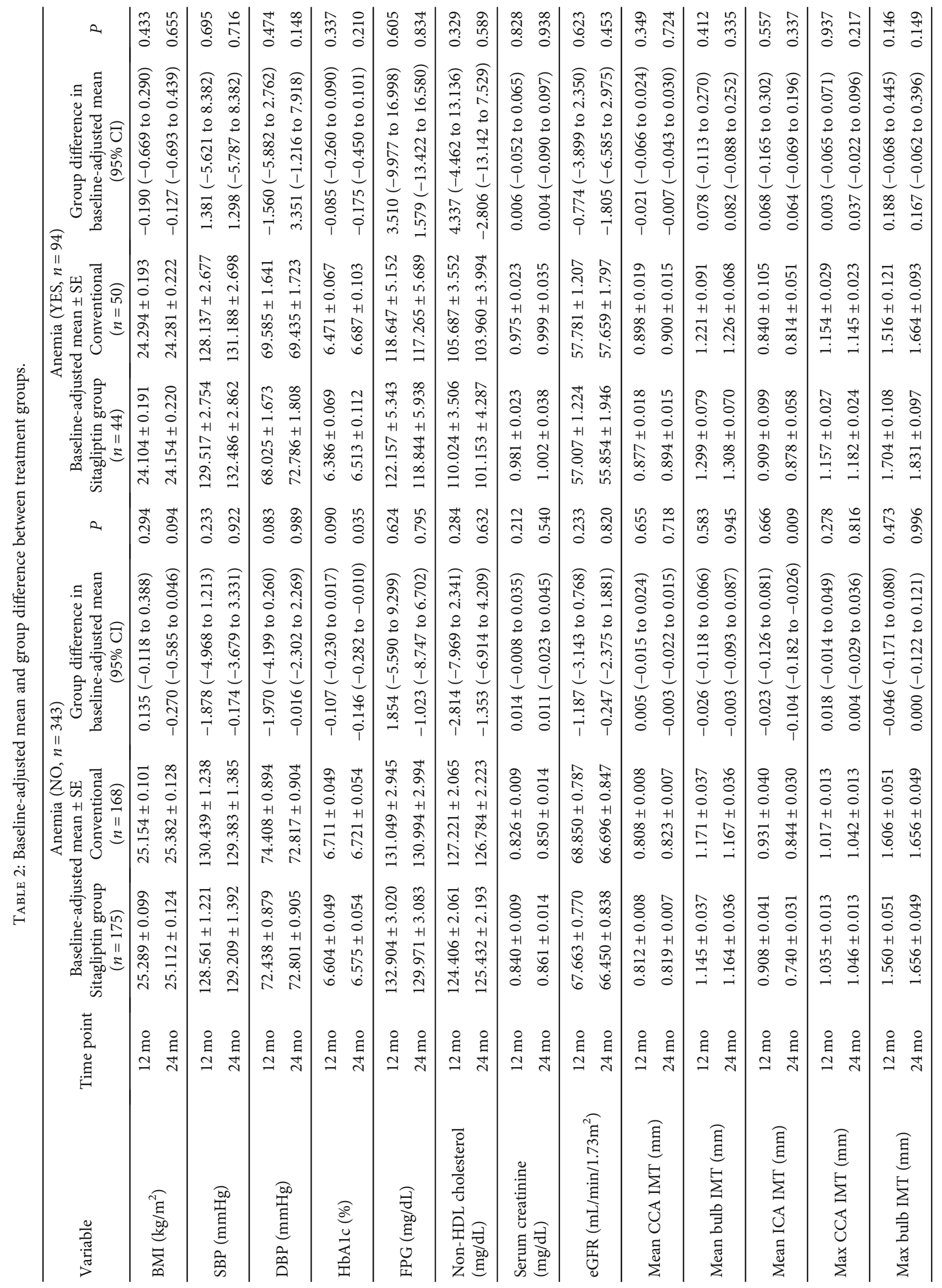




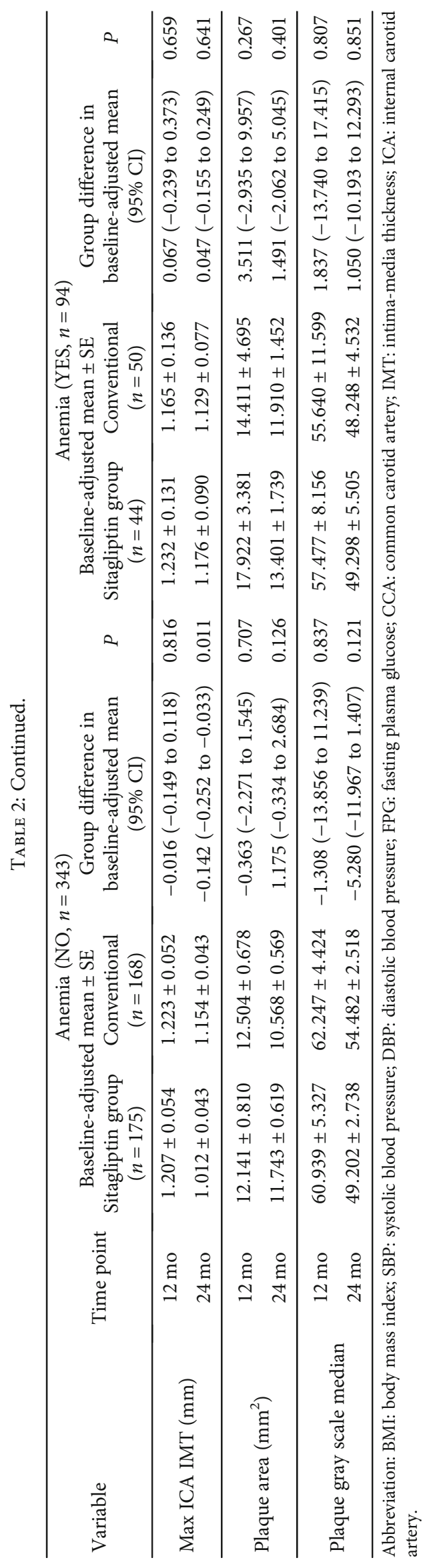




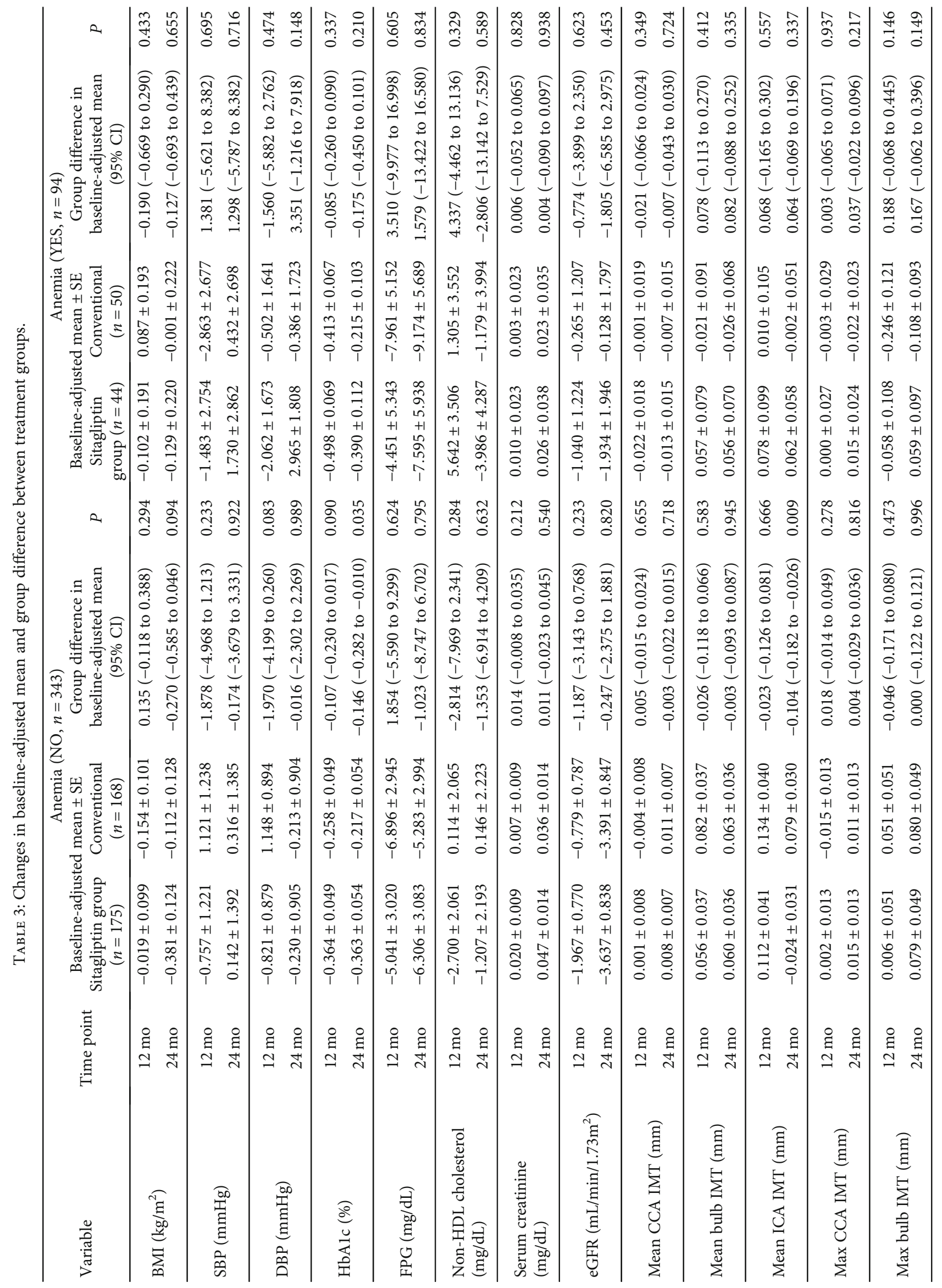




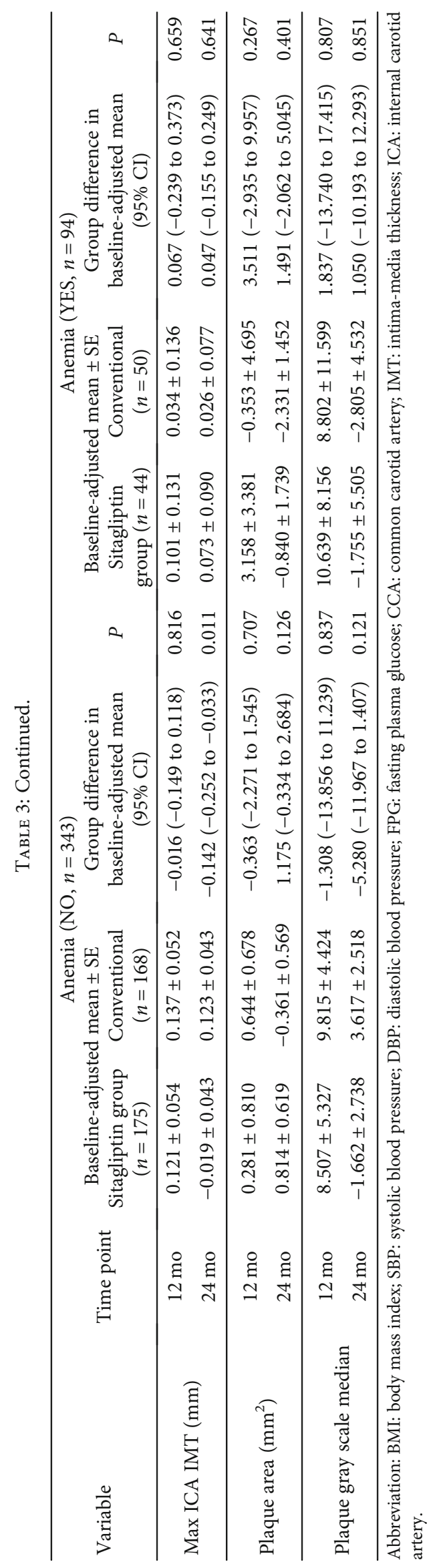


TABLE 4: Frequency of the use of antidiabetic and other agents.

\begin{tabular}{|c|c|c|c|c|c|c|c|}
\hline \multirow{2}{*}{ Variable } & \multirow{2}{*}{ Time point } & \multicolumn{3}{|c|}{ Anemia (NO) } & \multicolumn{3}{|c|}{ Anemia (YES) } \\
\hline & & Sitagliptin group & Conventional & $P$ & Sitagliptin group & Conventional & $P$ \\
\hline \multirow{3}{*}{ Sulfonylurea } & Baseline & $50(28.6 \%)$ & $42(25.0 \%)$ & 0.455 & $5(11.4 \%)$ & $10(20.0 \%)$ & 0.254 \\
\hline & $12 \mathrm{mo}$ & $35(21.3 \%)$ & $55(35.3 \%)$ & 0.006 & $4(10.8 \%)$ & $11(25.0 \%)$ & 0.102 \\
\hline & $24 \mathrm{mo}$ & $32(20.8 \%)$ & $51(34.2 \%)$ & 0.009 & $3(8.6 \%)$ & $10(23.3 \%)$ & 0.083 \\
\hline \multirow{3}{*}{ Metformin } & Baseline & $24(13.7 \%)$ & $19(11.3 \%)$ & 0.501 & $8(18.2 \%)$ & $13(26.0 \%)$ & 0.364 \\
\hline & $12 \mathrm{mo}$ & $30(18.3 \%)$ & $50(32.1 \%)$ & 0.004 & $8(21.6 \%)$ & $18(40.9 \%)$ & 0.064 \\
\hline & $24 \mathrm{mo}$ & $34(22.1 \%)$ & $50(33.6 \%)$ & 0.026 & $9(25.7 \%)$ & $18(41.9 \%)$ & 0.136 \\
\hline \multirow{3}{*}{$\alpha$-Glucosidase inhibitor } & Baseline & $57(32.6 \%)$ & $46(27.4 \%)$ & 0.294 & $14(31.8 \%)$ & $20(40.0 \%)$ & 0.410 \\
\hline & $12 \mathrm{mo}$ & $42(25.6 \%)$ & $61(39.1 \%)$ & 0.010 & $11(29.7 \%)$ & $23(52.3 \%)$ & 0.041 \\
\hline & $24 \mathrm{mo}$ & $35(22.7 \%)$ & $58(38.9 \%)$ & 0.002 & $10(28.6 \%)$ & $23(53.5 \%)$ & 0.027 \\
\hline \multirow{3}{*}{ Thiazolidinedione } & Baseline & $35(20.0 \%)$ & $36(21.4 \%)$ & 0.744 & $17(38.6 \%)$ & $17(34.0 \%)$ & 0.641 \\
\hline & $12 \mathrm{mo}$ & $25(15.2 \%)$ & $45(28.8 \%)$ & 0.003 & $13(35.1 \%)$ & $19(43.2 \%)$ & 0.461 \\
\hline & $24 \mathrm{mo}$ & $24(15.6 \%)$ & $47(31.5 \%)$ & 0.001 & $11(31.4 \%)$ & $15(34.9 \%)$ & 0.747 \\
\hline \multirow{3}{*}{ Glinide } & Baseline & $5(2.9 \%)$ & $15(8.9 \%)$ & 0.016 & $2(4.5 \%)$ & $4(8.0 \%)$ & 0.494 \\
\hline & $12 \mathrm{mo}$ & $1(0.6 \%)$ & $21(13.5 \%)$ & 0.0001 & $3(8.1 \%)$ & $4(9.1 \%)$ & 0.875 \\
\hline & $24 \mathrm{mo}$ & $1(0.6 \%)$ & $17(11.4 \%)$ & 0.0001 & $2(5.7 \%)$ & $4(9.3 \%)$ & 0.554 \\
\hline \multirow{3}{*}{ Statin } & Baseline & $133(76.0 \%)$ & $125(74.4 \%)$ & 0.732 & $33(75.0 \%)$ & $36(72.0 \%)$ & 0.743 \\
\hline & $12 \mathrm{mo}$ & $122(74.4 \%)$ & $113(72.4 \%)$ & 0.692 & $26(70.3 \%)$ & $30(68.2 \%)$ & 0.839 \\
\hline & $24 \mathrm{mo}$ & $114(74.0 \%)$ & $106(71.1 \%)$ & 0.573 & $25(71.4 \%)$ & $29(67.4 \%)$ & 0.704 \\
\hline \multirow{3}{*}{ Fibrate } & Baseline & $1(0.6 \%)$ & $3(1.8 \%)$ & 0.295 & $1(2.3 \%)$ & $0(0.0 \%)$ & 0.284 \\
\hline & $12 \mathrm{mo}$ & $1(0.6 \%)$ & $3(1.9 \%)$ & 0.291 & $1(2.7 \%)$ & $0(0.0 \%)$ & 0.273 \\
\hline & $24 \mathrm{mo}$ & $1(0.6 \%)$ & $3(2.0 \%)$ & 0.298 & $1(2.9 \%)$ & $0(0.0 \%)$ & 0.265 \\
\hline \multirow{3}{*}{ Angiotensin II receptor blocker } & Baseline & $98(56.0 \%)$ & $84(50.0 \%)$ & 0.266 & $31(70.5 \%)$ & $27(54.0 \%)$ & 0.102 \\
\hline & $12 \mathrm{mo}$ & $90(54.9 \%)$ & $80(51.3 \%)$ & 0.519 & $27(73.0 \%)$ & $24(54.5 \%)$ & 0.087 \\
\hline & $24 \mathrm{mo}$ & $87(56.5 \%)$ & $76(51.0 \%)$ & 0.338 & $27(77.1 \%)$ & $23(53.5 \%)$ & 0.030 \\
\hline \multirow{3}{*}{ Angiotensin-converting enzyme inhibitor } & Baseline & $20(11.4 \%)$ & $25(14.9 \%)$ & 0.344 & $6(13.6 \%)$ & $11(22.0 \%)$ & 0.293 \\
\hline & $12 \mathrm{mo}$ & $20(12.2 \%)$ & $23(14.7 \%)$ & 0.504 & $3(8.1 \%)$ & $9(20.5 \%)$ & 0.119 \\
\hline & $24 \mathrm{mo}$ & $16(10.4 \%)$ & $21(14.1 \%)$ & 0.325 & $3(8.6 \%)$ & $10(23.3 \%)$ & 0.083 \\
\hline
\end{tabular}

Number of patients in the NO anemia subgroup = baseline: 175 (Sita), 168 (Con); 12 months: 164 (Sita), 156 (Con); 24 months: 154 (Sita), 149 (Con). Number of patients in the anemia subgroup = baseline: 44 (Sita), 50 (Con); 12 months: 37 (Sita), 44 (Con); 24 months: 35 (Sita), 43 (Con). Data are expressed as number (\%).

frequency of noninvestigational drugs except glinide between the treatment groups in each subgroup. In each conventional group, the additional use of sulfonylureas, metformin, $\alpha$-glucosidase inhibitor, and thiazolidinedione increased during the 24 mo observation period. However, with the exception of metformin, there was no increase in drug use in each sitagliptin group (Table 4).

\section{Discussion}

The present study, a subgroup analysis using data obtained from the PROLOGUE study showed that sitagliptin treatment significantly inhibited the progression of mean and max ICA-IMT after 24 months observation period in the nonanemic subgroup, while the conventional treatment did not affect the ICA-IMT. But in the anemic subgroup, there was no significant difference in changes with CIMT between sitagliptin and conventional treatment groups. Although there was no significant difference in CCA-IMT between the treatment group in each subgroup, this analysis shows that sitagliptin treatment more effectively inhibited the progression of CIMT than conventional treatment in nonanemic T2DM patients. However, patients with T2DM and anemic do not seem to benefit from sitagliptin treatment.

Although anemia is a common complication in diabetic patients, it is often overlooked. And the relationship between anemia and ICA-IMT in patients undergoing sitagliptin treatment has not been described. This may be an important issue. Anemia is a strong risk factor for death in people with diabetes due to CVD [32-34]. Although many previous clinical studies have shown that there is a correlation between hemoglobin levels and subclinical atherosclerotic markers in patients with CVD or hypertension, however, the studies in patients with T2DM are rare. Therefore, elucidating the effect of hemoglobin levels on the development of atherosclerosis in patients with diabetes mellitus has great clinical 
significance. In the PROLOGUE study population, $21.3 \%$ of participants had baseline anemia events. If patients with T2DM and anemia benefit from sitagliptin treatment, that is important from a public health standpoint, as its use could slow ICA-IMT progression in nearly one-fourth of the T2DM patients with anemia.

The etiology of anemia in patients with diabetes has been previously reported and is considered to be multifactorial. In addition to diabetic nephropathy, the inflammation, nutritional deficiency, and hormone changes are all causes of anemia. Renal tubules are the main sites for the production of erythropoietin [38]. Systemic inflammation, reduced red cell survival, functional erythropoietin deficiency, and erythropoietin resistance caused by renal tubular dysfunction in patients with diabetes may lead to insufficient erythropoietin response $[31,39,40]$. In addition, absolute or relative (functional) iron deficiency is also a major cause of anemia in diabetes. In general, relative iron deficiency is more common and is closely related to the upregulation of inflammatory cytokines and impaired tissue responsiveness to erythropoietin. Previous studies have shown a link between anemia and CVD in diabetic patients. Even mild anemia can lead to serious complications in CVD, such as myocardial infarction [41, 42]. According to physiological theories, anemia affects tissue perfusion and oxygenation by a reduction in the oxygen-carrying ability of blood due to decreases in hemoglobin concentration and the change of blood apparent viscosity resulting from low hematocrit [43]. And blood viscosity can affect systemic vascular resistance, which leads to cardiovascular hemodynamics changes. Moreover, anemia has been suggested to be directly involved in tissue protection and regulation of cardiovascular homeostasis by affecting the atypical functions of erythrocytes. Erythrocytes are an important part of blood antioxidant capacity. Anemia significantly weakened the antioxidant system [44]. The activation of erythropoiesis can lead to the decrease of oxidative stress, which in turn prevents atherosclerotic progression [45]. Atherosclerosis is one of the main factors contributing to an increase of CIMT [46]. Anemia may lead to endothelial dysfunction and decrease the level of nitric oxide (NO) by reducing endothelial shear stress, which in turn accelerates atherosclerosis by increasing the oxidation of low-density lipoprotein (LDL) [47-50]. Ganidagli et al. found that the patient (LH group) with the highest hemoglobin variability had the highest CIMT (LN: $0.601 \mathrm{~mm}, \mathrm{LH}: 0.744 \mathrm{~mm}$, NH: $0.604 \mathrm{~mm}, p<0.001$ ) [51]. Dogan and Citak found that low levels of hemoglobin were associated with increased CIMT in children with $\beta$-thalassemia major [52]. These findings support that changes in hemoglobin levels may lead to arterial remodeling. In the post hoc analysis, we also found that the baseline CIMT parameters of the anemic subgroup were higher than those of the nonanemic subgroup.

Our results suggest that patients with diabetes may be more significant to see the benefits of sitagliptin treatment before the development of anemia. For patients with anemia, it may be difficult to see the benefit of sitagliptin treatment. There is biologic plausibility of the findings that patients with T2DM and nonanemia can benefit from sitagliptin treatment. Anemia is associated with increased progression of atherosclerosis, increased carotid artery intimal-medial thickness, and cardiac hypertrophy [53]. The pathophysiological process of atherosclerosis in patients with anemia may be more advanced. These patients may not be able to benefit from sitagliptin treatment. In addition, the control of blood glucose helps to improve the tissue characteristics of carotid artery plaque. Although the decrease in HbAlc levels in the anemic subgroup was greater in the sitagliptin group than that in the conventional group, we consider that most patients have achieved relatively good blood glucose control and strict management of risk factors. Therefore, the progression of CIMT may be partially affected by anemia, although the additional effect of sitagliptin on the progression of CIMT was not shown in patients with risk factors for anemia. As an adjunct treatment for statins, angiotensin-converting enzyme inhibitors and angiotensin II receptor blockers, it seems to have a unique or additive antiatherosclerotic effects in T2DM patients without anemia. Previous studies have confirmed that statins and angiotensinconverting enzyme inhibitors can reduce the development of atherosclerosis in T2DM patients [54, 55]. The T2DM patients without anemia risk factors should choose more appropriate drugs for prevention and treatment. Furthermore, we speculate that a higher frequency of the use of angiotensin II receptor blocker and thiazolidinedione in anemia subgroup may be part of the reason for this result. Because the inhibitory effect of angiotensin II receptor blocker and thiazolidinedione on CIMT has also been confirmed [56,57], this may mask or diminish the effectiveness of investigational drugs. In the PROLOGUE study, patients inevitably took other drugs, and it was difficult to rule out their effects. However, we reiterate that the real intention of the present study as an exploratory subgroup analysis is to explore whether sitagliptin can have an additional effect on the CIMT process. Our subgroup study did not find that sitagliptin slowed the progression of CIMT in patients with T2DM and anemia. The precise reasons are not clear. Tanaka et al. pointed out that T2DM patients with or without previous CV events were recruited in the PROLOGUE study [58], while other studies investigating the effects of DPP-4 inhibitors only recruited T2DM patients without events $[24,25]$.

Our study has the following limitations. First, it is a post hoc subanalysis using data obtained from the PROLOGUE study. The analysis was not part of the original statistical plan. Therefore, the result needs to be treated with caution. Second, other noninvestigational drugs such as antidiabetic, antihyperlipidemic, and antihypertensive may influence the CIMT progression, although the baseline drug therapies were almost matched in this post hoc analysis. Third, the sample size may not be sufficient to detect significant differences in CIMT variables between treatment groups in the anemia subgroup. In order to confirm our findings, it is necessary to design a prespecified study with a large sample size.

\section{Conclusions}

In this post hoc study of PROLOGUE study data, the patients with T2DM and nonanemia can obtain better antiatherosclerotic effects in the sitagliptin treatment group compared 
with the conventional group. Since this finding may be attributed to sampling variability, therefore, it would be premature to conclude that sitagliptin treatment significantly inhibited CIMT progression. Further research is needed.

\section{Data Availability}

Data and materials are available upon the Dryad Digital Repository. (https://doi.org/10.5061/dryad.qt743).

\section{Ethical Approval}

The ethics committee of the participating agency approved the research protocol.

\section{Consent}

All participants should provide written informed consent before data collection.

\section{Disclosure}

The role of the Funder/Sponsor: Funding organizations are not involved in the design and conduct of research; the collection, management, analysis, and interpretation of data; the preparation, review, or approval of manuscripts; and the decision to submit manuscripts for publication.

\section{Conflicts of Interest}

On behalf of all authors, the corresponding author states that there is no conflict of interest.

\section{Authors' Contributions}

ZRL was assigned on the study conception and design, data collection, statistical analyses, and drafting of the manuscript. LJC was assigned on the critical revisions to the manuscript. GSM was assigned on critical revisions to the manuscript. All authors read and approved the final manuscript.

\section{Acknowledgments}

The authors gratefully thank Junichi Oyama, Toyoaki Murohara, Masafumi Kitakaze, Tomoko Ishizu, Yasunori Sato, Kazuo Kitagawa, Haruo Kamiya, Masayoshi Ajioka, Masaharu Ishihara, Kazuoki Dai, Mamoru Nanasato, Masataka Sata, Koji Maemura, Hirofumi Tomiyama, Yukihito Higashi, Kohei Kaku, Hirotsugu Yamada, Munehide Matsuhisa, Kentaro Yamashita, Yasuko K. Bando, Naoki Kashihara, Shinichiro Ueda, Teruo Inoue, Atsushi Tanaka, Koichi Node, and PROLOGUE Study Investigators for sharing their data. The authors are grateful to the staff of the Institute of Cardiovascular Disease at Southeast University. The authors gratefully thank Huijuan Zheng for polishing the manuscript. Funding for the study was provided by the National Nature Science Foundation of China (Grant No. 81770231 and No. 81270203); the Natural Science Foundation of Jiangsu (Grant No. BK20161436); the Jiangsu Provincial Key Medical Discipline (Laboratory No. ZDXKA2016023); and the Jiangsu Provincial Key Research and Development Program (Grant No. BE2016785).

\section{References}

[1] L. Whiteley, S. Padmanabhan, D. Hole, and C. Isles, "Should diabetes be considered a coronary heart disease risk equivalent?: results from 25 years of follow-up in the Renfrew and Paisley survey," Diabetes Care, vol. 28, no. 7, pp. 1588-1593, 2005.

[2] L. Lundby-Christensen, T. P. Almdal, B. Carstensen, L. Tarnow, and N. Wiinberg, "Carotid intima-media thickness in individuals with and without type 2 diabetes: a reproducibility study," Cardiovascular Diabetology, vol. 9, no. 1, p. 40, 2010.

[3] D. H. O'Leary, J. F. Polak, R. A. Kronmal, T. A. Manolio, G. L. Burke, and S. K. Wolfson Jr., "Carotid-artery intima and media thickness as a risk factor for myocardial infarction and stroke in older adults. Cardiovascular Health Study Collaborative Research Group," The New England Journal of Medicine, vol. 340, no. 1, pp. 14-22, 1999.

[4] E. de Groot, G. K. Hovingh, A. Wiegman et al., "Measurement of arterial wall thickness as a surrogate marker for atherosclerosis," Circulation, vol. 109, 23 Supplement 1, pp. III33-III38, 2004.

[5] H. N. Hodis, W. J. Mack, L. LaBree et al., "The role of carotid arterial intima-media thickness in predicting clinical coronary events," Annals of Internal Medicine, vol. 128, no. 4, pp. 262269, 1998

[6] T. Mita, N. Katakami, T. Shiraiwa et al., "Changes in carotid intima-media thickening in patients with type 2 diabetes mellitus: subanalysis of the Sitagliptin Preventive Study of IntimaMedia Thickness Evaluation," Journal of Diabetes Investigation, vol. 8, no. 2, pp. 254-255, 2017.

[7] S. A. Peters, D. E. Grobbee, and M. L. Bots, "Carotid intimamedia thickness: a suitable alternative for cardiovascular risk as outcome?," European Journal of Cardiovascular Prevention and Rehabilitation, vol. 18, no. 2, pp. 167-174, 2011.

[8] Q. F. Han, L. Wu, T. Li, X. Y. Meng, and H. C. Yao, "There is a link between carotid intima media thickness and coronary artery disease: it might be inflammation," International Journal of Cardiology, vol. 203, pp. 1144-1145, 2016.

[9] Y. Huang, W. Li, L. Dong, R. Li, and Y. Wu, "Effect of statin therapy on the progression of common carotid artery intimamedia thickness: an updated systematic review and metaanalysis of randomized controlled trials," Journal of Atherosclerosis and Thrombosis, vol. 20, no. 1, pp. 108-121, 2013.

[10] D. F. Geng, D. M. Jin, W. Wu, C. Fang, and J. F. Wang, "Effect of alpha-glucosidase inhibitors on the progression of carotid intima-media thickness: a meta-analysis of randomized controlled trials," Atherosclerosis, vol. 218, no. 1, pp. 214-219, 2011.

[11] X. Song, H. Jia, Y. Jiang et al., “Anti-atherosclerotic effects of the glucagon-like peptide-1 (GLP-1) based therapies in patients with type 2 diabetes mellitus: a meta-analysis," Scientific Reports, vol. 5, no. 1, article 10202, 2015.

[12] S. G. Chrysant and G. S. Chrysant, "Clinical implications of cardiovascular preventing pleiotropic effects of dipeptidyl peptidase-4 inhibitors," The American Journal of Cardiology, vol. 109, no. 11, pp. 1681-1685, 2012. 
[13] G. P. Fadini and A. Avogaro, "Cardiovascular effects of DPP-4 inhibition: beyond GLP-1," Vascular Pharmacology, vol. 55, no. 1-3, pp. 10-16, 2011.

[14] M. Terasaki, M. Nagashima, K. Nohtomi et al., "Preventive effect of dipeptidyl peptidase- 4 inhibitor on atherosclerosis is mainly attributable to incretin's actions in nondiabetic and diabetic apolipoprotein E-null mice," PLoS One, vol. 8, no. 8, article e70933, 2013.

[15] Y. Terawaki, T. Nomiyama, T. Kawanami et al., "Dipeptidyl peptidase-4 inhibitor linagliptin attenuates neointima formation after vascular injury," Cardiovascular Diabetology, vol. 13, no. 1, p. 154, 2014.

[16] T. Hirano and Y. Mori, "Anti-atherogenic and antiinflammatory properties of glucagon-like peptide-1, glucosedependent insulinotropic polypepide, and dipeptidyl peptidase- 4 inhibitors in experimental animals," Journal of Diabetes Investigation, vol. 7, Supplement 1, pp. 80-86, 2016.

[17] J. Matsubara, S. Sugiyama, K. Sugamura et al., "A dipeptidyl peptidase-4 inhibitor, des-fluoro-sitagliptin, improves endothelial function and reduces atherosclerotic lesion formation in apolipoprotein E-deficient mice," Journal of the American College of Cardiology, vol. 59, no. 3, pp. 265-276, 2012.

[18] F. Vittone, A. Liberman, D. Vasic et al., "Sitagliptin reduces plaque macrophage content and stabilises arteriosclerotic lesions in Apoe $e^{(--)}$mice," Diabetologia, vol. 55, no. 8, pp. 2267-2275, 2012.

[19] N. Satoh-Asahara, Y. Sasaki, H. Wada et al., "A dipeptidyl peptidase-4 inhibitor, sitagliptin, exerts anti-inflammatory effects in type 2 diabetic patients," Metabolism, vol. 62, no. 3, pp. 347-351, 2013.

[20] A. J. Tremblay, B. Lamarche, C. F. Deacon, S. J. Weisnagel, and P. Couture, "Effects of sitagliptin therapy on markers of lowgrade inflammation and cell adhesion molecules in patients with type 2 diabetes," Metabolism, vol. 63, no. 9, pp. 11411148, 2014.

[21] B. M. Scirica, D. L. Bhatt, E. Braunwald et al., "Saxagliptin and cardiovascular outcomes in patients with type 2 diabetes mellitus," The New England Journal of Medicine, vol. 369, no. 14, pp. 1317-1326, 2013.

[22] W. B. White, C. P. Cannon, S. R. Heller et al., "Alogliptin after acute coronary syndrome in patients with type 2 diabetes," The New England Journal of Medicine, vol. 369, no. 14, pp. 13271335, 2013.

[23] J. B. Green, M. A. Bethel, P. W. Armstrong et al., "Effect of sitagliptin on cardiovascular outcomes in type 2 diabetes," The New England Journal of Medicine, vol. 373, no. 3, pp. 232242, 2015.

[24] T. Mita, N. Katakami, H. Yoshii et al., "Alogliptin, a dipeptidyl peptidase 4 inhibitor, prevents the progression of carotid atherosclerosis in patients with type 2 diabetes: the study of preventive effects of alogliptin on diabetic atherosclerosis (SPEAD-A)," Diabetes Care, vol. 39, no. 1, pp. 139-148, 2016.

[25] T. Mita, N. Katakami, T. Shiraiwa et al., "Sitagliptin attenuates the progression of carotid intima-media thickening in insulin-treated patients with type 2 diabetes: the Sitagliptin Preventive Study of Intima-Media Thickness Evaluation (SPIKE): a randomized controlled trial," Diabetes Care, vol. 39, no. 3, pp. 455-464, 2016.

[26] N. Katakami, T. Mita, Y. Irie et al., "Effect of sitagliptin on tissue characteristics of the carotid wall in patients with type 2 diabetes: a post hoc sub-analysis of the sitagliptin preven- tive study of intima-media thickness evaluation (SPIKE)," Cardiovascular Diabetology, vol. 17, no. 1, p. 24, 2018.

[27] V. Lambadiari, G. Pavlidis, F. Kousathana et al., "Effects of 6month treatment with the glucagon like peptide-1 analogue liraglutide on arterial stiffness, left ventricular myocardial deformation and oxidative stress in subjects with newly diagnosed type 2 diabetes," Cardiovascular Diabetology, vol. 17, no. 1, p. 8, 2018.

[28] M. C. Thomas, R. J. MacIsaac, C. Tsalamandris, D. Power, and G. Jerums, "Unrecognized anemia in patients with diabetes: a cross-sectional survey," Diabetes Care, vol. 26, no. 4, pp. 1164-1169, 2003.

[29] M. C. Thomas, R. J. MacIsaac, C. Tsalamandris et al., "The burden of anaemia in type 2 diabetes and the role of nephropathy: a cross-sectional audit," Nephrology, Dialysis, Transplantation, vol. 19, no. 7, pp. 1792-1797, 2004.

[30] M. J. Sarnak, H. Tighiouart, G. Manjunath et al., "Anemia as a risk factor for cardiovascular disease in the Atherosclerosis Risk in Communities (ARIC) study," Journal of the American College of Cardiology, vol. 40, no. 1, pp. 27-33, 2002.

[31] K. J. Craig, J. D. Williams, S. G. Riley et al., "Anemia and diabetes in the absence of nephropathy," Diabetes Care, vol. 28, no. 5, pp. 1118-1123, 2005.

[32] M. C. Thomas, C. Tsalamandris, R. J. MacIsaac, and G. Jerums, "The epidemiology of hemoglobin levels in patients with type 2 diabetes," American Journal of Kidney Diseases, vol. 48, no. 4, pp. 537-545, 2006.

[33] P. T. Vlagopoulos, H. Tighiouart, D. E. Weiner et al., “Anemia as a risk factor for cardiovascular disease and all-cause mortality in diabetes: the impact of chronic kidney disease," Journal of the American Society of Nephrology, vol. 16, no. 11, pp. 3403-3410, 2005.

[34] S. I. McFarlane, M. O. Salifu, J. Makaryus, and J. R. Sowers, "Anemia and cardiovascular disease in diabetic nephropathy," Current Diabetes Reports, vol. 6, no. 3, pp. 213-218, 2006.

[35] J. Oyama, T. Murohara, M. Kitakaze et al., "The effect of sitagliptin on carotid artery atherosclerosis in type 2 diabetes: the PROLOGUE randomized controlled trial," PLoS Medicine, vol. 13, no. 6, article e1002051, 2016.

[36] The Committee of the Japan Diabetes Society on the Diagnostic Criteria of Diabetes Mellitus, Y. Seino, K. Nanjo et al., "Report of the committee on the classification and diagnostic criteria of diabetes mellitus," Journal of Diabetes Investigation, vol. 1, no. 5, pp. 212-228, 2010.

[37] World Health Organization, Haemoglobin concentrations for the diagnosis of anaemia and assessment of severity, World Health Organization, 2011.

[38] T. Basturk, Y. Altuntas, A. Kurklu, L. Aydin, N. Eren, and A. Unsal, "Urinary $\mathrm{N}$-acetyl B glucosaminidase as an earlier marker of diabetic nephropathy and influence of low-dose perindopril/indapamide combination," Renal Failure, vol. 28, no. 2, pp. 125-128, 2006.

[39] M. C. Thomas, C. Tsalamandris, R. Macisaac, and G. Jerums, "Functional erythropoietin deficiency in patients with type 2 diabetes and anaemia," Diabetic Medicine, vol. 23, no. 5, pp. 502-509, 2006.

[40] M. C. Thomas, "Anemia in diabetes: marker or mediator of microvascular disease?," Nature Clinical Practice Nephrology, vol. 3, no. 1, pp. 20-30, 2007. 
[41] J. P. Bassand, R. Afzal, J. Eikelboom et al., "Relationship between baseline haemoglobin and major bleeding complications in acute coronary syndromes," European Heart Journal, vol. 31, no. 1, pp. 50-58, 2010.

[42] M. Rousseau, R. T. Yan, M. Tan et al., "Relation between hemoglobin level and recurrent myocardial ischemia in acute coronary syndromes detected by continuous electrocardiographic monitoring," The American Journal of Cardiology, vol. 106, no. 10, pp. 1417-1422, 2010.

[43] A. C. Guyton and J. E. Hall, Textbook of Medical Physiology, Saunders, Philadelphia, PA, USA, 1986.

[44] T. Grune, O. Sommerburg, and W. G. Siems, "Oxidative stress in anemia," Clinical Nephrology, vol. 53, Supplement 1, pp. S18-S22, 2000.

[45] M. Noguchi-Sasaki, Y. Sasaki, Y. Matsuo-Tezuka et al., "Reduction of a marker of oxidative stress with enhancement of iron utilization by erythropoiesis activation following epoetin beta pegol administration in iron-loaded $\mathrm{db} / \mathrm{db}$ mice," International Journal of Hematology, vol. 103, no. 3, pp. 262273, 2016.

[46] J. F. Polak and D. H. O'Leary, "Carotid intima-media thickness as surrogate for and predictor of CVD," Global Heart, vol. 11, no. 3, pp. 295-312.e3, 2016, e293.

[47] C. M. Boulanger, N. Amabile, A. P. Guerin et al., "In vivo shear stress determines circulating levels of endothelial microparticles in end-stage renal disease," Hypertension, vol. 49, no. 4, pp. 902-908, 2007.

[48] J. Davignon and P. Ganz, "Role of endothelial dysfunction in atherosclerosis," Circulation, vol. 109, no. 23, Supplement 1, pp. III27-III32, 2004.

[49] J. Malyszko, "Mechanism of endothelial dysfunction in chronic kidney disease," Clinica Chimica Acta, vol. 411, no. 19-20, pp. 1412-1420, 2010.

[50] K. U. Eckardt, "Anaemia in end-stage renal disease: pathophysiological considerations," Nephrology, Dialysis, Transplantation, vol. 16, Supplement 7, pp. 2-8, 2001.

[51] S. E. Ganidagli, O. Altunoren, E. Erken et al., "The relation between hemoglobin variability and carotid intima-media thickness in chronic hemodialysis patients," International Urology and Nephrology, vol. 49, no. 10, pp. 1859-1866, 2017.

[52] M. Dogan and E. C. Citak, "The evaluation of carotid intimamedia thickness in children with beta-thalassaemia major," Cardiology in the Young, vol. 22, no. 1, pp. 79-83, 2012.

[53] F. Metivier, S. J. Marchais, A. P. Guerin, B. Pannier, and G. M. London, "Pathophysiology of anaemia: focus on the heart and blood vessels," Nephrology, Dialysis, Transplantation, vol. 15, Supplement 3, pp. 14-18, 2000.

[54] N. Fang, W. Han, D. Gong, Zou Chen, and Y. Fan, “Atorvastatin treatment for carotid intima-media thickness in Chinese patients with type 2 diabetes: a meta-analysis," Medicine, vol. 94, no. 44, article e1920, 2015.

[55] N. Hosomi, K. Mizushige, H. Ohyama et al., "Angiotensinconverting enzyme inhibition with enalapril slows progressive intima-media thickening of the common carotid artery in patients with non-insulin-dependent diabetes mellitus," Stroke, vol. 32, no. 7, pp. 1539-1545, 2001.

[56] H. Ono, S. Minatoguchi, K. Watanabe et al., "Candesartan decreases carotid intima-media thickness by enhancing nitric oxide and decreasing oxidative stress in patients with hypertension," Hypertension Research, vol. 31, no. 2, pp. 271-279, 2008.
[57] E. M. Lonn, H. C. Gerstein, P. Sheridan et al., "Effect of ramipril and of rosiglitazone on carotid intima-media thickness in people with impaired glucose tolerance or impaired fasting glucose: STARR (STudy of Atherosclerosis with Ramipril and Rosiglitazone)," Journal of the American College of Cardiology, vol. 53, no. 22, pp. 2028-2035, 2009.

[58] A. Tanaka, H. Yoshida, M. Nanasato et al., "Sitagliptin on carotid intima-media thickness in type 2 diabetes patients receiving primary or secondary prevention of cardiovascular disease: a subgroup analysis of the PROLOGUE study," International Journal of Cardiology, vol. 271, pp. 331-335, 2018. 